\title{
Rocketborne instrument to search for infrared emission from baryonic dark matter in galactic halos
}

James J. Bock, Mitsunobu Kawada, Andrew E. Lange, Toshio Matsumoto, Kazunori Uemizu, et al.

James J. Bock, Mitsunobu Kawada, Andrew E. Lange, Toshio Matsumoto, Kazunori Uemizu, Toyoki Watabe, S. A. Yost, Giovanni G. Fazio, William J. Forrest, Judith L. Pipher, Stephan D. Price, "Rocketborne instrument to search for infrared emission from baryonic dark matter in galactic halos," Proc. SPIE 3354, Infrared Astronomical Instrumentation, (21 August 1998); doi: $10.1117 / 12.317239$

Event: Astronomical Telescopes and Instrumentation, 1998, Kona, HI, United States 


\title{
Rocket-borne instrument to search for infrared emission from baryonic dark matter in galactic halos
}

\author{
James J. Bock ${ }^{a}$, Mitsunobu Kawada ${ }^{b}$, Andrew E. Lange ${ }^{c}$, Toshio Matsumoto ${ }^{b}$, \\ Kazunori Uemizu ${ }^{\text {bd }}$, Toyoki Watabe ${ }^{d}$, Sarah A. Yost ${ }^{c}$, Giovanni G. Fazio, \\ William J. Forrest ${ }^{f}$, Judith L. Pipher ${ }^{f}$, and Stephan D. Price ${ }^{g}$ \\ ajet Propulsion Laboratory, Pasadena, CA 91109 \\ ${ }^{b}$ Institute of Space and Astronautical Science, Kanagawa, 229-8510, Japan \\ ${ }^{c}$ California Institute of Technology, Pasadena, CA 91125 \\ ${ }^{\mathrm{d}}$ Nagoya University, Nagoya 464-8602, Japan \\ ${ }^{\mathrm{e}}$ Smithsonian Astrophysical Observatory, Cambridge, MA 02138 \\ ${ }^{f}$ University of Rochester, Rochester, NY 14627 \\ ${ }^{\mathrm{g}}$ Air Force Research Laboratory, Hanscom AFB, MA 01731
}

\begin{abstract}
We describe the design and performance of the Near Infrared Telescope Experiment (NITE), a rocket-borne instrument designed to search for infrared emission from baryonic dark matter in the halos of nearby edge-on spiral galaxies. A $256 \mathrm{x}$ $256 \mathrm{InSb}$ array at the focus of a $16.5 \mathrm{~cm}$ liquid-helium-cooled telescope achieves near-background-limited sensitivity in a 3.5 - $5.5 \mu \mathrm{m}$ waveband where the local foreground from zodiacal emission is at a minimum. This experiment represents the first scientific application of a low-background infrared InSb array, a precursor to the InSb arrays intended for SIRTF, in a spaceborne observation. We describe the flight performance of the instrument and preliminary scientific results from an observation of NGC 4565.
\end{abstract}

Keywords: Infrared astronomy, InSb, galactic halos, baryonic matter

\section{INTRODUCTION}

The predominant mass in a typical spiral galaxy is known to exist in a form of non-luminous dark matter ${ }^{1}$. Although dark matter is thought to dominate the total mass of the universe, the nature of the dark matter is unknown and is one of the most important unsolved problems in astrophysics. A significant fraction of galactic dark matter may reside in the form of baryons. The upper limit on the density of baryons from standard big-bang nucleosynthesis $\left(\Omega_{b}<0.1 \mathrm{~h}^{-2}\right)$ does not seriously constrain the nature of dark matter surrounding galaxies. Recent evidence points to a substantial baryonic component to galactic dark matter. Gravitational microlensing experiments have detected the existence of lensing objects (massive compact halo objects, or 'machos') in the halo of our galaxy with sub-solar mass. Based on the most recent results of the MACHO project', the optical depth towards the LMC is now estimated to be $\tau=2.9-0.9^{+1.4} \times 10^{-7}$, fully $\sim 50 \%$ of the value expected for a standard halo composed entirely of machos. The most probable macho mass of $0.5_{-0.2}^{+0.3}$ solar masses is model dependent and thus highly uncertain.

The nature of the baryonic objects detected in the microlensing experiments remains unknown, but may plausibly be in the form of stellar objects with high mass-to-light ratio such as low-mass stars, brown dwarfs or white dwarfs. Low-mass stars with mass greater than $\sim 0.08$ solar masses undergo fusion. Brown dwarfs, "failed stars" with mass under the hydrogen-

Further author information -

Email: James.J.Bock@jpl.nasa.gov; Telephone: (818)-354-0715; Fax: (626)-584-9929

G.G.F.: gfazio@cfa.harvard.edu Tel: (617)-495-7458

M.K.: kawada@astro.isas.ac.jp Tel: 81-427-86-7201

T.M.: matsumo@astro.isas.ac.jp Tel: 81-427-86-7201

S.D.P.: price@plh.af.mil Tel: (617)-377-4552

T.W.: watabe@u-phys.nagoya-u.ac.jp Tel: 81-52-789-5247

W.J.F.: forrest@sherman.pas.rochester.edu Tel: (716)-275-4343

A.E.L.: ael@astro.caltech.edu Tel: (626)-395-6887

J.L.P.: jlpipher@sherman.pas.rochester.edu Tel: (716)-275-4402

K.U.: uemizu@astro.isas.ac.jp Tel: 81-427-86-7201

S.A.Y.: say@ astro.caltech.edu (626)-395-2020 
burning limit, heat by gravitational collapse and slowly radiate away their energy over a time-scale comparable to the age of the universe. White dwarfs would constitute the low-mass stellar remnants formed by a primordial burst of star formation in the galactic halo.

Attempts to detect the abundance of low-mass stars through local surveys at optical wavelengths have not reached a clear consensus yet, although the bulk of the evidence may be against abundant production of low-mass stars ${ }^{3}$. Near-Infrared surveys (DENIS, 2MASS) will further probe the local abundance of low-mass stellar objects. Recently Matthews et $a l^{4}$ have reported observations of a faint $\left(\mathrm{L}_{\mathrm{Bol}}=6.4 \times 10^{-6} \mathrm{~L}_{\text {solar }}\right)$ brown dwarf, identifiable by strong methane absorption features in its spectrum. The spectrum of low-metallicity, primordial low-mass stars and brown dwarfs is currently the subject of active theoretical work ${ }^{5}$, but is expected to be much different than that of objects with solar metallicity. White dwarfs with sub-solar mass, remnants of an early burst of star formation in the halo, are also a plausible candidate for the detected massive compact halo objects. White dwarfs slowly cool and should now have a temperature of $\sim 3000 \mathrm{~K}$, radiating predominately in the infrared ${ }^{6}$. Estimates of the mass-to-light ratio for galactic halos consisting entirely of low-mass stars, brown dwarfs, and white dwarfs depend strongly on the mass and age of the stellar objects.

The Near-Infrared Telescope Experiment (NITE) tests the hypothesis that galactic dark matter resides in the form of dim, low-mass stars or dwarfs by creating a deep near-infrared image of a nearby edge-on spiral galaxy. The density of dark matter surrounding a spiral galaxy is inferred by measuring the rotational velocity as a function of galactic radius. The presence of low-mass stars or dwarfs in the halo gives rise to an emission component that varies as $\sim 1 / \mathrm{r}$ in the near infrared image. This component must be distinguished from the emission from stars in the galactic bulge and core which varies as $\sim 1 / \mathrm{r}^{3}$.

Experiments to detect extended emission from the halo component of an edge-on spiral galaxy may be conducted from the ground at optical and near-infrared wavelengths. Initial searches in the near-infrared ${ }^{7-8}$ set upper limits on the halo brightness, constraining the mass-to-light ratio of the halo to be $M / L_{K}>(40-60) M_{\text {solar }} / L_{\text {solar }}$. More recently, ground-based measurements ${ }^{9-10}$ report the existence of a red $(R-K \sim 3.5)$ luminous $\left(M / L_{R} \sim 100\right)$ halo around NGC 5907. In constrast to the ground-based experiments, NITE observes at 3.5 - 5.5 $\mu \mathrm{m}$ where the local foreground from zodiacal emission is at a minimum, and at longer wavelengths where emission from cool, red objects can be expected to be more prominent. NITE images infrared halo emission over a wide $1.2^{\circ} \times 1.2^{\circ}$ field. The wide field is useful to distinguish a true halo component from a thick disk component or from the tidally digested remnants of gas-rich dwarf galaxies.

Redshifted starlight from distant galaxies is predicted to produce an infrared extragalactic background. DIRBE has mapped the sky multiple times in 10 bands between $1 \mu \mathrm{m}$ and $250 \mu \mathrm{m}$ in an effort to separate this background from the local zodiacal foreground. Based on galaxy counts at K-band ${ }^{11}$ the infrared background must exist within an order of magnitude of the estimated DIRBE sky residuals ${ }^{12}$. Whether DIRBE is successful or not in detecting the diffuse background light in the near-infrared, the logical succession to DIRBE is to probe the infrared sky on a finer angular scale. In the process of observing the target galaxy NITE observes several square degrees of blank sky. NITE measures the zodiacal brightness with a signal-to-noise ratio in excess of 100 per pixel. Estimated surface brightness fluctuations from unresolved infrared galaxies, based on the galactic evolution models of Franceshini ${ }^{13}$, are detectable by NITE and dominate over the fluctuations from stars for observations at high galactic latitudes. Determination of the confusion limit in this wavelength band is of significance for future near-infrared observations from space.

\section{INSTRUMENT DESCRIPTION}

NITE consists of a near-infrared camera mounted at the focus of a liquid-helium-cooled $16.5 \mathrm{~cm}$ Gregorian telescope. The detector is a low-background 256 × 256 Indium-Antimonide (InSb) array which achieves $<15 \mathrm{e}^{-}$read noise and $<1 \mathrm{e}^{-/} / \mathrm{s}$ dark current in laboratory operation. NITE is the first science experiment to use such an array, originally developed for SIRTF ${ }^{14}$, outside the earth's atmosphere. The arrangement of the telescope, cooled baffle tube, filter, shutter, calibration lamp, and focal plane array is shown in Fig. 1.

The $16.5 \mathrm{~cm}$ doubly-folded Gregorian telescope is made entirely of Beryllium in order to minimize image distortion due to differential contraction and deformation during cooling. An internal image of the primary mirror located at the position of the fourth mirror element is heavily baffled to prevent the detector from viewing the primary skirt, reducing the stray light response. The telescope provides an unvignetted field of $1.2^{\circ} \times 1.2^{\circ}$, with the angular size subtended by a single 


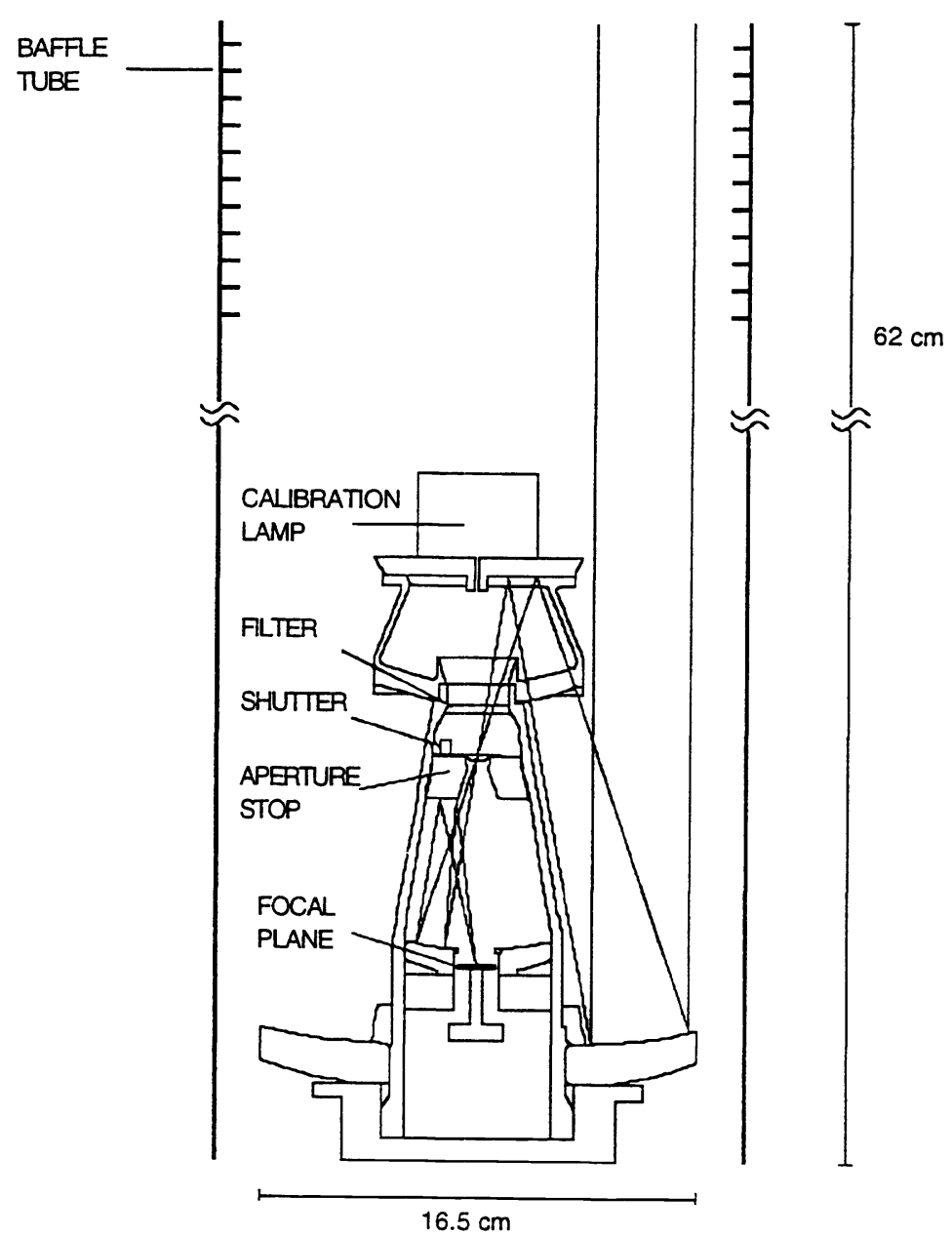

Figure 1: The optics of NITE, consisting of a Beryllium $16.5 \mathrm{~cm}$ folded Gregorian telescope, infrared filter, shutter, calibration lamp, and $256 \times 256 \mathrm{InSb}$ focal plane array. The telescope is housed within a cooled baffle tube to reduce response to off-axis radiation.

pixel of 17 " $\times 17$ ". The optics are designed to minimize geometric aberrations over the wide field of view, with an rms spot diameter < 20" out to the edge of the field of view and a maximum rms spot size of $38^{\prime \prime}$ at the corner of the field of view. A $3.5 \mu \mathrm{m}$ long wavelength pass infrared filter, placed in the optical path above the $\mathrm{f} / 1.54$ focus of the primary mirror, is located to prevent radiation reflected by the detector from re-reflecting on the filter and creating a ghost image. The long wavelength edge of the $3.5-5.5 \mu \mathrm{m}$ passband is formed by the detector cutoff. We estimate that the total optical efficiency of the filter and detector is greater than $70 \%$.

A cold shutter located inside the telescope housing interrupts the beam at the position of the prime focus and enables a measurement of the detector dark current. The bistable shutter is operated by applying a pulse of electrical current in a solenoid to switch positions. The shutter is based on a modified design of the shutter used for ISO $^{15}$. A calibration source located inside the telescope housing is used to monitor the responsivity of the detector array in order to track pixel-to-pixel gain fluctuations to assist in removing point sources. The calibration source consists of a lamp and integrating sphere to provide uniform illumination of the focal plane. The detector is thermally isolated on a stainless steel support tube and heated to the optimal operating temperature. The telescope is housed inside a cooled black vaned baffle tube designed to reject offaxis radiation. The detector array, telescope, baffle tube, shutter, and calibration lamp are housed in a super-critical helium cryostat which maintains a temperature of $5.2 \mathrm{~K}$ in zero gravity.

We multiply sample the InSb focal plane array to reduce the detector read noise, sampling the array continuously at $3.8 \mathrm{~Hz}$. Although coadded sampling provides slightly improved noise performance and greatly reduced telemetry rate, we selected continuous sampling to simplify the flight electronics and to allow for maximum flexibility in post-flight data 
analysis. In the laboratory we typically achieve a read noise of $25 \mathrm{e}^{-}$for a $10 \mathrm{~s}$ integration, consistent with the expected noise for combining 38 frames. Including read noise and photon noise with a flight photocurrent of $150 \mathrm{e}^{-} / \mathrm{s}$ we thus expect a total read noise of $45 \mathrm{e}^{-}$, within a factor of 1.2 of background-limited performance.

To prevent the detector from viewing emission from warm portions of the cryostat, several black baffles had to be placed near the focal plane. Using a dark, light-tight cover over the cold baffle tube, we are able to achieve a detector current $<2 \mathrm{e}^{-} / \mathrm{s}$, which includes both dark current and any residual photocurrent. In flight configuration, the ejectable lid gives a measurable background due to thermal emission from the shields and the imperfect light seal around the cooled baffle tube. Before launch, with the ejectable lid in place, the detector current was $<20 \mathrm{e}^{-/ \mathrm{s}}$ with the shutter open and $<2 \mathrm{e}^{-} / \mathrm{s}$ with the shutter closed.

The instrument is housed inside the skin of the rocket as shown in Fig. 2. The skin is a vacuum section with a sealed bulkhead towards the nose side and a sealing shutter door on the aft side. The shutter door opens during the flight to initiate observations. The skin section is first evacuated and then purged with dry nitrogen gas before launch to reduce the quantity of water vapor and other contaminants, and seals on reentry to protect the instrument for recovery. To further reduce the

\section{Stowed}

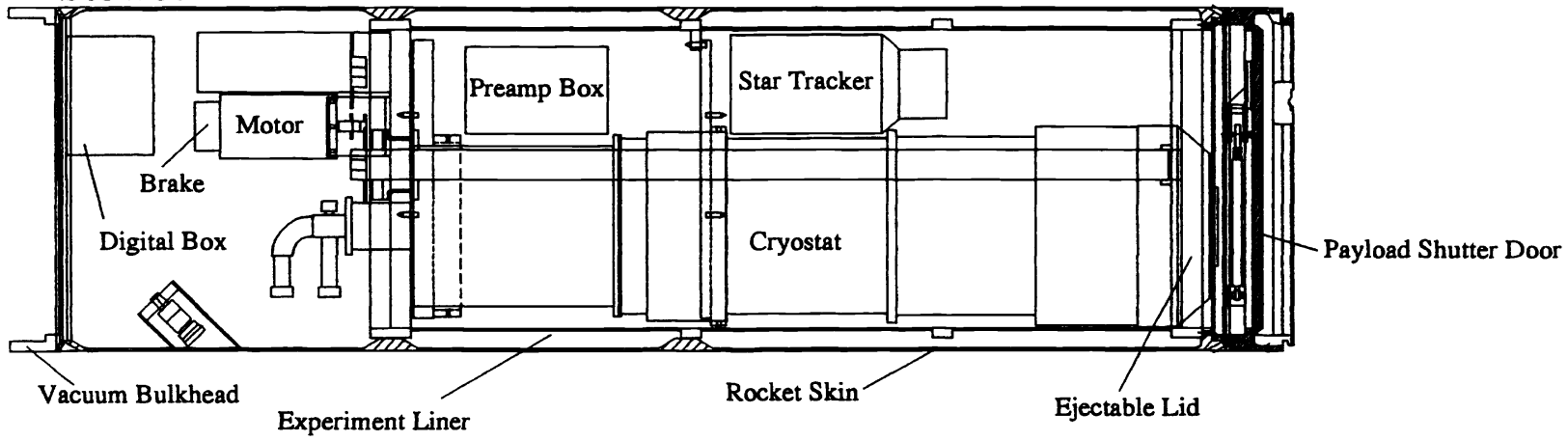

Extended

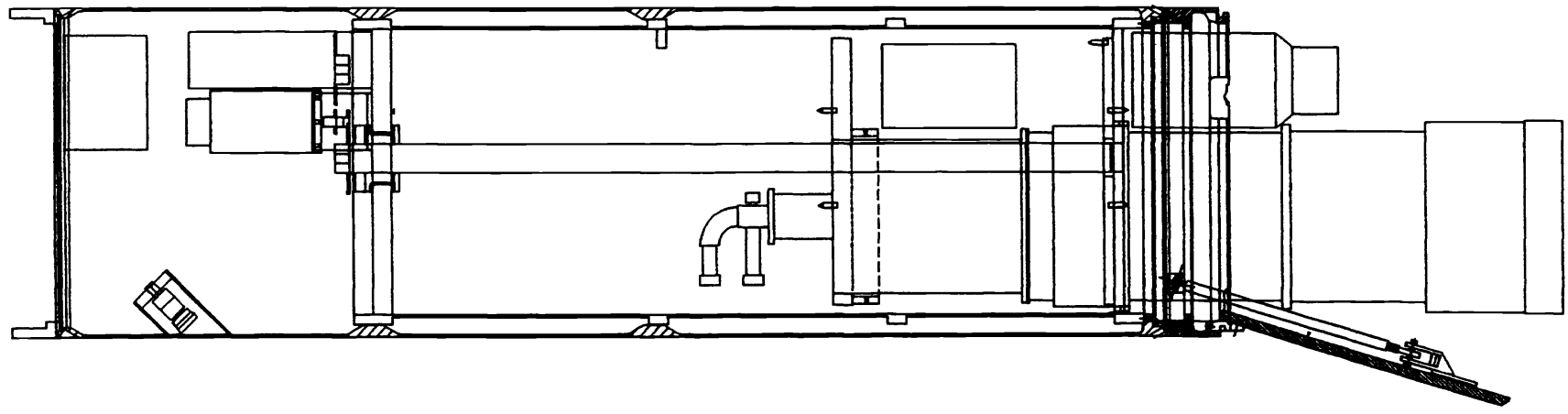

Figure 2: Overview of the instrument housed inside the vacuum-tight rocket skin. The telescope, detector, and cooled baffle tube are housed in a supercritical He cryostat. The star tracker and preamplification electronics mount directly on the cryostat. The cryostat mounts to two plates threaded into twin shafts. Operating the motor turns the shafts so that the cooled baffle tube may extend past the end of the open, ambient-temperature shutter door. The lid placed over the cryostat may by released by cutting a single wire with a pyrotechnic wire cutter, releasing clamps and activating springs which eject the lid out of the field of view. Retractable cables electrically connect the preamplifier box and the digital electronics box. 
possibility of rocket-borne contamination, the booster is separated from the payload by a high velocity separation system while rotating at $1.35 \mathrm{~Hz}$ to induce a coning motion in the booster, insuring that it does not tumble or pass the payload. The payload is despun to zero rotation with a special gas despin system developed by the NASA sounding rocket program. The NASA portions of the instrument are all purged with dry nitrogen gas for 24 hours before the launch.

The camera is extremely sensitive to thermal emission from room-temperature surfaces, which are many orders of magnitude brighter than the astrophysical sky at these wavelengths. Therefore a translation mechanism is used to extend the cold baffle tube past the shutter door during the observations so that the telescope does not view any portion of the warm payload. The translation mechanism mounts on a liner which bolts to the rocket skin at a single point near the location of the shutter door in order to remove the effect of differential expansion caused by frictional heating of the skin during ascent. The cryostat is mounted on twin threaded shafts, and a motor turns the shafts to extend and retract the instrument. A star tracker is coaligned with the telescope field of view and mounts to the cryostat to remove any pointing offset induced by the translation mechanism. The instrument is pointed by a NASA Mark VI attitude control system using nitrogen gas thrusters. In the stowed position, the instrument is secured from motion in the radial direction by locating pins and from motion in the translational direction by the threaded shafts and an electro-mechanical brake.

The specifications of NITE are indicated in Table 1 based on the performance of the detector during the flight: 150 $\mathrm{e}^{-} / \mathrm{s}$ photocurrent, a total readout noise of $45 \mathrm{e}^{-}$(which includes read noise and photon noise), a reset interval of $10 \mathrm{~s}$, and a total observation time of $290 \mathrm{~s}$, including $60 \mathrm{~s}$ spent in pointing overhead, $20 \mathrm{~s}$ in viewing the internal calibration source, $10 \mathrm{~s}$ in viewing calibration stars, and $200 \mathrm{~s}$ observing NGC 4565. The large format of the $256 \mathrm{x} 256$ InSb array provides an important advantage in mapping speed, offsetting the large advantage in integration time available with the $32 \mathrm{x} 32$ arrays on ISOCAM. Transients and non-linearities in the ISOCAM focal plane arrays, which can in practice limit the sensitivity to surface brightness in deep integrations, have negligible effect in the NITE InSb focal plane array.

Table 1: Instrument Specifications

\begin{tabular}{|c|c|}
\hline Field of view & $1.2^{\circ} \times 1.2^{\circ}$ \\
Pixel size & $17^{\prime \prime} \times 17^{\prime \prime}$ \\
Array Format & $256 \times 256 \mathrm{InSb}$ \\
Waveband & $3.5-5.5 \mu \mathrm{m}$ \\
Read noise & $25 \mathrm{e}^{-}$ \\
Photon noise & $40 \mathrm{e}^{-}$ \\
Pixel sensitivity (vI $, 1 \sigma, 10 \mathrm{~s})$ & $8 \times 10^{-13} \mathrm{~W} \mathrm{~cm}^{-2} \mathrm{sr}^{-1}$ \\
Observation time on NGC 4565 & $200 \mathrm{~s}$ \\
\hline
\end{tabular}

\section{FLIGHT PERFORMANCE}

NITE was launched on a NASA Terrier Black Brant sounding rocket at 22:56, 28 May 1997 from White Sands Missile Range to observe the nearby edge-on spiral galaxy NGC 4565. The flight events before the observations were as follows: Terrier stage burnout at $6.2 \mathrm{~s}$, Black Brant burnout at $44.4 \mathrm{~s}$, despin to $1.35 \mathrm{~Hz}$ at $81 \mathrm{~s}$, payload separation at $86 \mathrm{~s}$ followed by full despin, shutter door open at $94 \mathrm{~s}$, translation mechanism extension at $100 \mathrm{~s}$, cryostat lid ejection at $125 \mathrm{~s}$, and guide star acquisition with the star tracker from $135-170 \mathrm{~s}$ in order to provide accurate absolute pointing for the science observations. The payload achieved an apogee of $345 \mathrm{~km}$ and provided $290 \mathrm{~s}$ of science observation time. The observations terminate at $472 \mathrm{~s}$ when the telescope retracts and the shutter door closes, followed by re-entry into the atmosphere and deployment of the parachute for recovery.

The sequence of events during the flight relating to the science instrument are shown in Fig. 3. Bright calibration stars $S 1$ and $S 2$ are observed for $5 \mathrm{~s}$ at the beginning of the observation and at the end of the observation, respectively, to characterize the point spread function of the telescope and to calibrate the response of the instrument. The edge-on spiral galaxy NGC 4565 is observed by placing it on a quadrant of the detector for $23.5 \mathrm{~s}$ allowing for two $10 \mathrm{~s}$ exposures of the sky and a brief observation of the flux from the calibration lamp superposed on the sky. The galaxy is then moved to each quadrant of the detector in succession. The pointing is offset by $\sim 10$ pixels and the observation cycle is repeated. The 


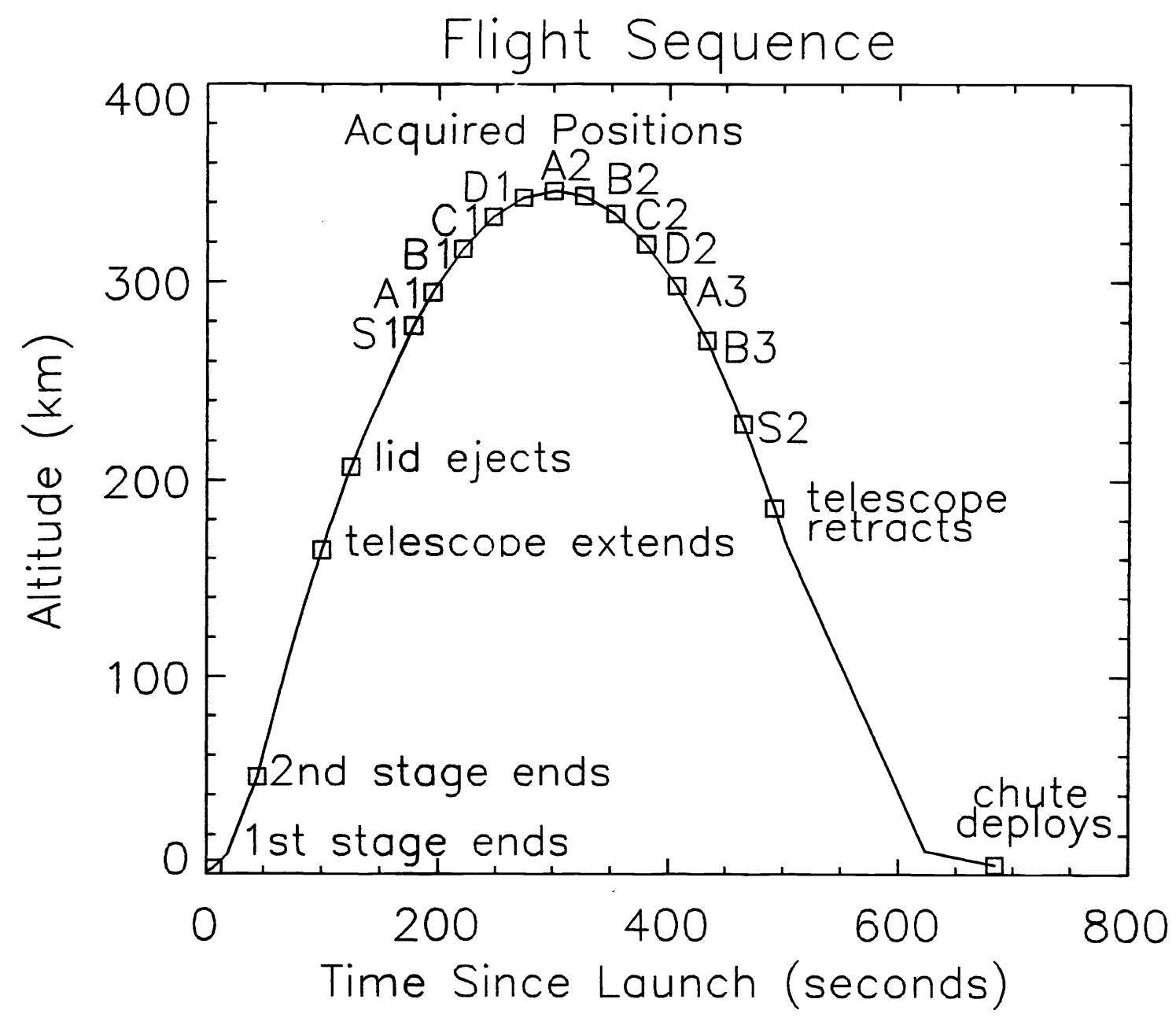

Figure 3: Sequence of selected flight events during the sounding rocket flight plotted as a function of altitude and time. Observations of NGC 4565 take place between $195 \mathrm{~s}$ and $455 \mathrm{~s}$ when the altitude exceeds $250 \mathrm{~km}$.

observation pattern is displayed in Fig. 4.

Our observation strategy allows simultaneous observation of the target and blank sky for accurate removal of the zodiacal foreground. The galaxy is observed with multiple pixels to reduce pixel-to-pixel flat field variations. Because a large fraction of the array views blank sky at any time, algorithms to remove the halo from the zodiacal foreground can be systematically tested, over the same number of pixels, by using the blank sky data. For example, the differenced blank sky data can be used to check for drift residuals or other systematic effects. Mapping a large area of blank sky allows us to probe for systematic error arising from residual structure in the zodiacal foreground.

The telescope, cryostat, cold shutter, calibrator lamp, cryostat lid and payload shutter door all functioned normally during the flight. The shutter closed during the ascent portion of the flight to monitor the detector dark current, and remained closed during the early pointing maneuvers so that the detector did not view bright emission from the earth when the telescope was pointed near the horizon. The shutter opened during the science observations and closed again during the recovery sequence. The focal plane array and readout electronics operated at near-background limited performance. We estimate the read noise in flight by differencing 2 sequential exposures on the same region of sky, giving a read noise of $45 \mathrm{e}^{-}$over a $10 \mathrm{~s}$ 


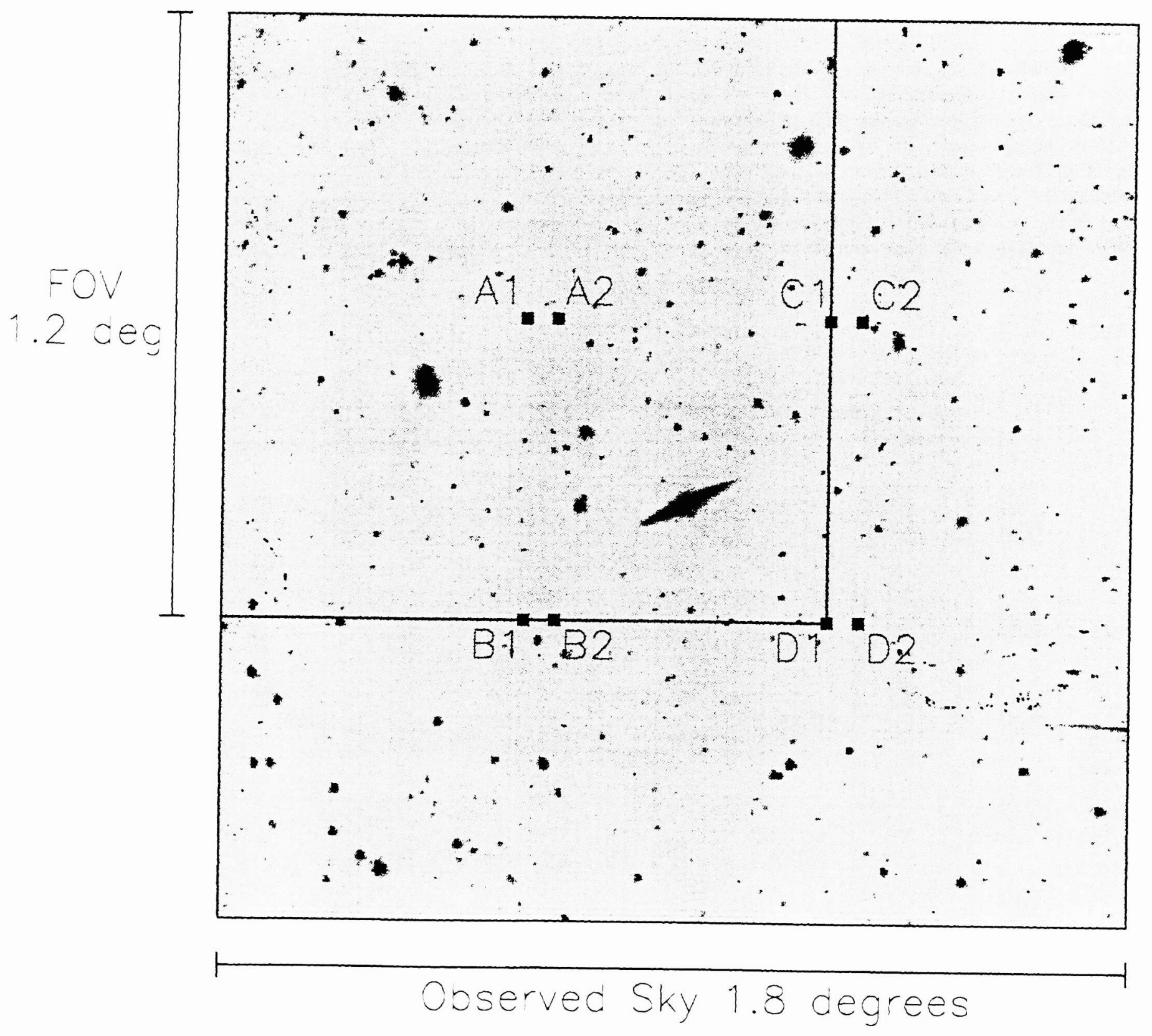

Figure 4: Observation scheme used to observe the edge-on spiral galaxy places the target in a quadrant of the array for two $10 \mathrm{~s}$ exposures and a short observation of the calibration lamp superposed on the sky brightness. The position of the center of the $1.2^{\circ} \times 1.2^{\circ}$ field of view is denoted by the labeled squares. The field of view is then shifted by steps of $0.6^{\circ}$, placing the target in each quadrant of the array in sequence and mapping $1.8^{\circ} \times 1.8^{\circ}$ of sky. Upon returning to the original quadrant, the position of the target is slightly offset to allow different pixels to observe the same sky, and the cycle is repeated. 
integration. One column of the array, sharing a common readout amplifier had an abnormal offset level and gain, and showed intermittent excess noise. We attribute this problem to a wire bond on the focal plane array that was damaged during vibration testing in the preflight integration. Although it was not possible to access the array before the launch, the wire bond was repaired after the flight and the array again functioned normally. The faulty wire bond did not seriously degrade the science goals of the experiment.

The science targets were acquired by the NASA Mark VI attitude control system, capable of pointing the instrument within 5' absolute accuracy and $<5$ " jitter. All of the science targets were acquired successfully except the last $40 \mathrm{~s}$ of observations of the galaxy and the final calibration star when the attitude control system became unstable. The sky brightness viewed by the instrument, $260 \mathrm{nW} / \mathrm{m}^{2} \mathrm{sr}$ based on a preliminary calibration using the first calibration star S1, is consistent with the brightness expected from zodiacal light. During the useful observation period of NGC 4565 when the attitude control system functioned properly, the absolute brightness observed by the instrument remained stable to within $\pm 5 \%$ as shown in Fig. 5. Thus any emission component from the atmosphere or the instrument was both small and stable. The science observations take place with the telescope pointed near the zenith, so earthshine does not illuminate any portion of the

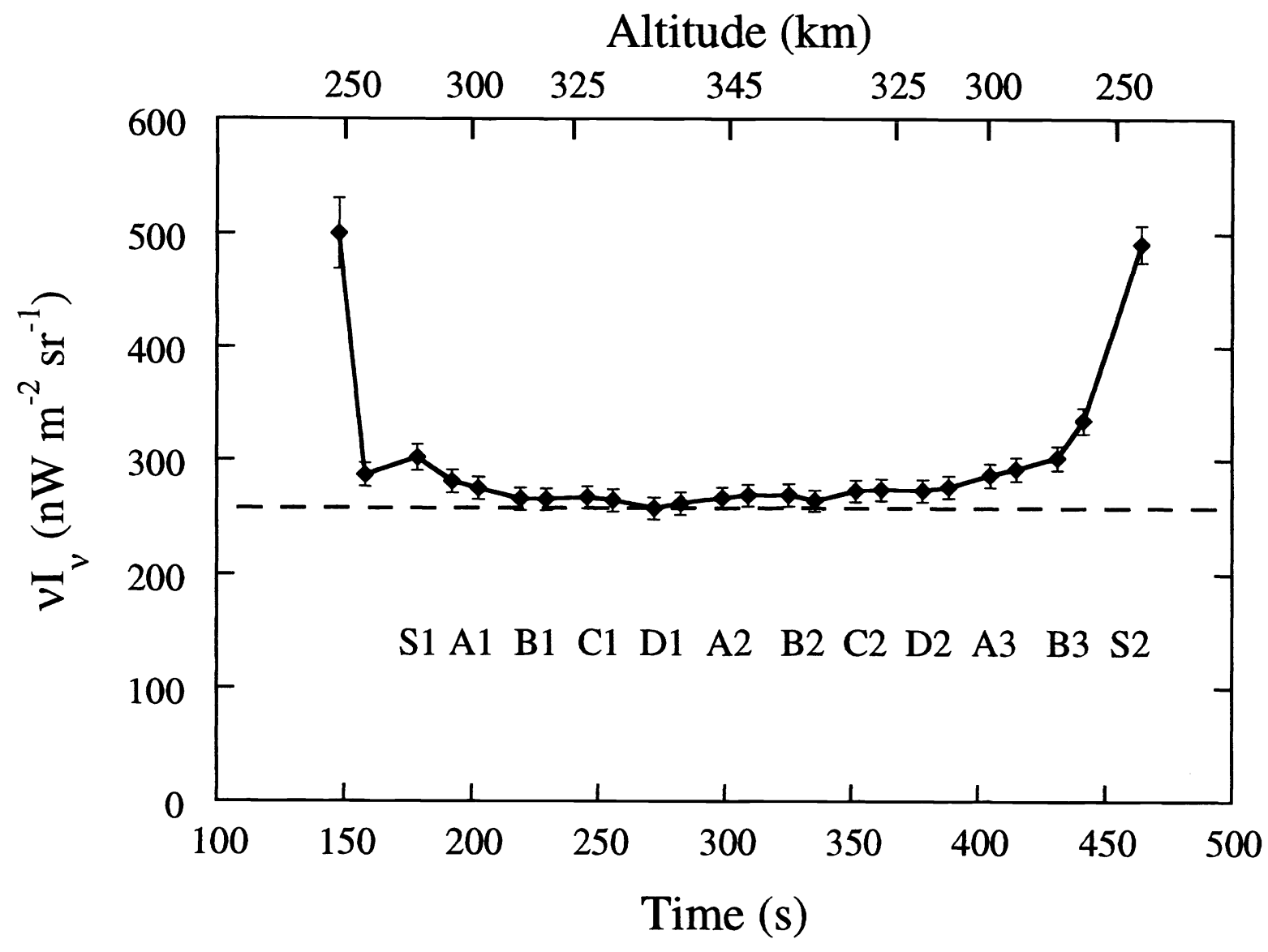

Figure 5: Absolute sky brightness observed by the detector as a function of time during the observations. The slight increase at the beginning and end of the observations is most likely due to atmospheric emission from the earth. The minimum surface brightness obtained during the flight at an ecliptic latitude of $27^{\circ}, 260 \mathrm{nW} / \mathrm{m}^{2} \mathrm{sr}$, is comparable to the minimum zodiacal brightness reported by DIRBE ${ }^{12}$ at the south ecliptic pole in this waveband, $150 \mathrm{nW} / \mathrm{m}^{2} \mathrm{sr}$. 
baffle tube. The translation mechanism failed to retract at the end of the flight due to a problem with a microswitch position sensor, causing the translation mechanism, star tracker, and baffle tubes to be damaged on recovery. However, the telescope, focal plane array, electronics, and cryostat did not sustain damage and functioned normally after the flight.

\section{PRELIMINARY RESULTS}

Extended emission surrounding the edge-on spiral galaxy NGC 4565 is evident in the raw images. The point spread function (PSF) of the instrument, characterized in the observations of stars in the field, S1, and the bright guide stars, may account for some of the extended emission. However, we convolved the measured PSF with the measured bright core and bulge of NGC 4565 and found that the PSF accounts for $<20 \%$ of the extended flux of the galaxy for $r>6^{\prime}$. The performance of the instrument during the flight and preliminary science results may be reported based on the following analysis. We can reduce the observations of the galaxy by forming the quantity

$$
S_{i j}=\left(A_{i j}-B_{i j}+C_{i j}-D_{i j}\right) \text {, }
$$

where $A_{i j}, B_{i j}, C_{i j}$, and $D_{i j}$ denote a 10 s exposure of the galaxy in each of the 4 positions shown in Fig. 4 . The resulting

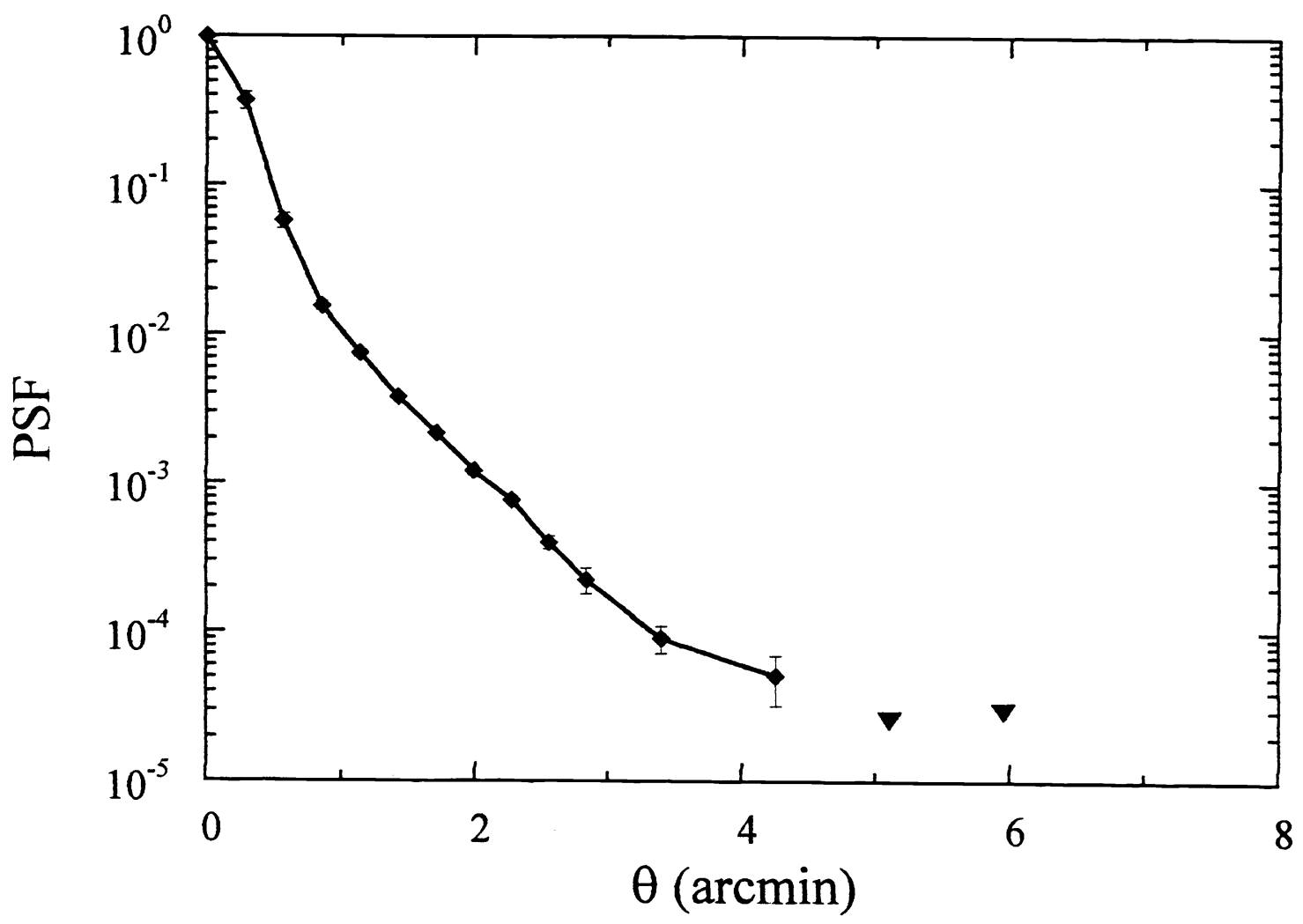

Figure 6: The point spread function of the instrument measured from bright stars in the field of view. Upper limits $(2 \sigma)$ are shown as diamonds. For $\mathrm{r}<3^{\prime}$ the point spread function falls at least as rapidly as $\mathrm{r}^{-5}$. Further analysis on the saturated guide stars observed early in the flight should allow us to measure the PSF beyond 4'. 


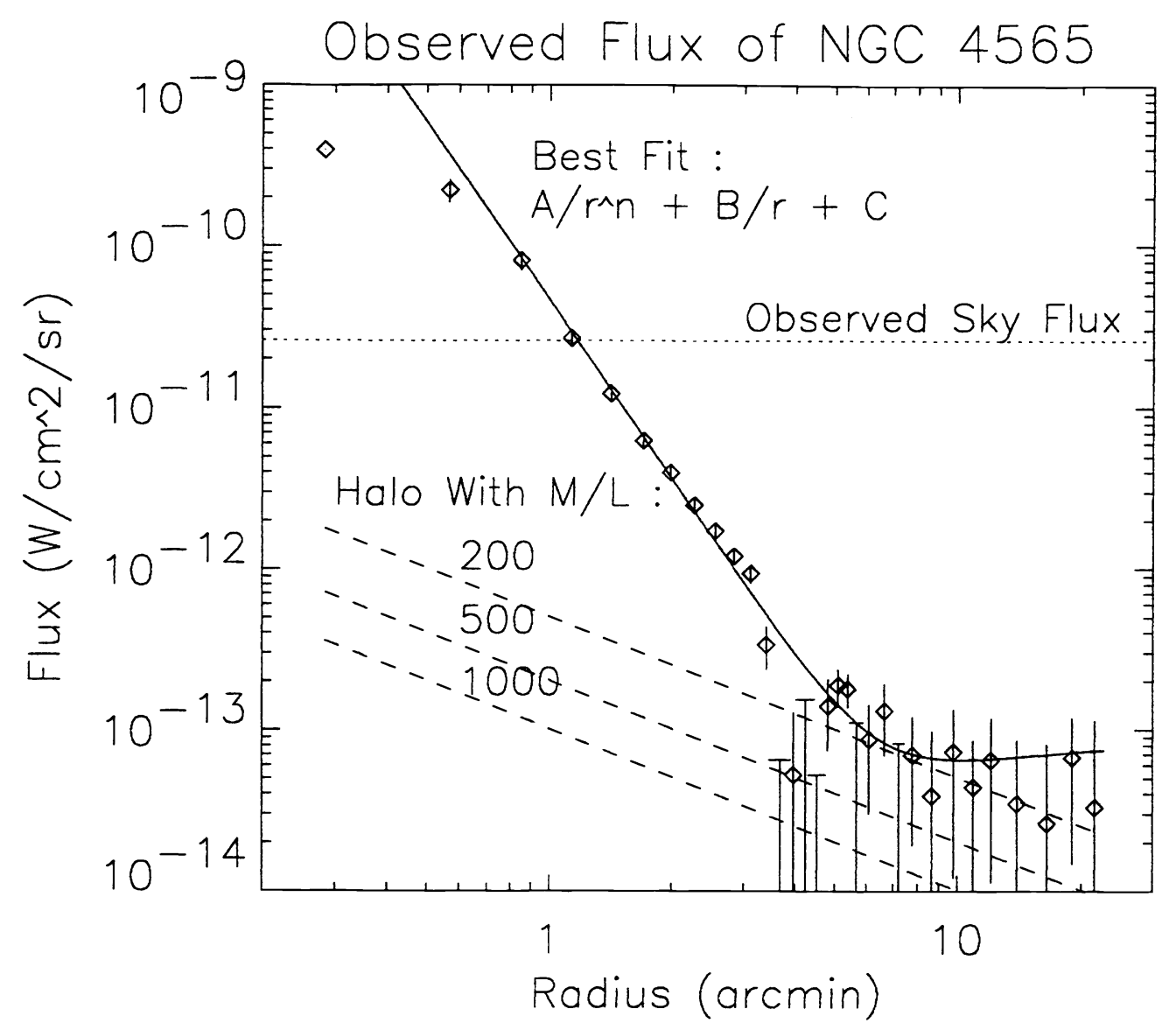

Figure 7: Measured brightness as a function of radius from the centroid of the galaxy. The brightness is measured by coadding pixels within annuli. The best fit to the data, excluding the innermost two points, is given by the solid line. The expected emission from the halo is shown as dashed lines for different mass-to-light ratios. The small offset term described in the text dominates the fit at large radii.

image $S_{\mathrm{ij}}$ has 4 subimages of the galaxy, two positive and two negative, which we align using the centroid of the galaxy and coadd after clipping for stars and removing bad pixels. Finally we coadd the 4 resulting images for each $S_{i j}$ to coadd all 16 useful exposures. The advantage of this approach is that constant dark current and sky components are subtracted, removing the need to accurately account for the flat field response of the array. The disadvantages are that it increases the number of stars to remove due to negative stars in the off positions, and limits the analysis of the halo to a region $36^{\prime} \times 36^{\prime}$.

We plot the flux of the galaxy in annuli centered about the galaxy as shown in Fig. 6. The width of the annuli vary, being small to give higher resolution at smaller radii, and large to give more statistical significance at larger radii. A wedge is removed aligned with galactic disk to reduce emission from the disk and its extension. The measured surface brightness is fit to the 4-parameter function

$$
\mathrm{Ar}^{-\mathrm{n}}+\mathrm{Br}^{-1}+\mathrm{C} \text {, }
$$

where $\mathrm{A}=(4.64 \pm 0.37) \times 10^{-11} \mathrm{~W} \mathrm{~cm}^{-2} \mathrm{sr}^{-1}, \mathrm{n}=3.64 \pm 0.15, \mathrm{~B}=(-3.6 \pm 2.4) \times 10^{-13} \mathrm{~W} \mathrm{~cm}^{-2} \mathrm{sr}^{-1}$ and $\mathrm{C}=(9.1 \pm 2.0) \times 10^{-14} \mathrm{~W}$ $\mathrm{cm}^{-2} \mathrm{sr}^{-1}$, and $\mathrm{r}$ is measured in units of arc minutes. These values include only the sources of statistical error. The $\mathrm{Ar}^{-\mathrm{n}}$ term is included to fit the emission component to the bulge and disk and their extensions. The constant term $\mathrm{C}$ is needed to remove a small residual offset which is $0.35 \%$ of the absolute sky brightness. This term may arise from residual time-dependent emission from the atmosphere or the payload during the observations, a drift in the detector dark current, or structure in the 
zodiacal emission. The term $\mathrm{Br}^{-1}$ is included to probe for the existence of near-infrared emission from the halo with density profile $\rho \sim r^{-2}$, but no halo component is detected. Full analysis of the data including a discussion of systematic errors is in preparation $^{16}$.

\section{CONCLUSIONS}

We have developed an instrument to measure the extended infrared brightness surrounding an edge-on spiral galaxy in a search for emission from baryonic galactic dark matter. The instrument achieves near-background-limited performance by multiply sampling a low-background InSb focal plane array. Our scan strategy and the sensitivity and stability of the instrument allow us to successfully probe surface brightness levels 100 times fainter than the zodiacal background.

\section{ACKNOWLEDGMENTS}

We would like to thank the payload team involved in our 1997 flight for their dedicated efforts. This research was supported by a grant from the Caltech President's Fund, NASA grant NAG5-4079, and by Grant-in-Aid 06402002 for Scientific Research from the Japanese Ministry of Education, Science, Sports, and Culture.

\section{REFERENCES}

1. Ashmann, K. M., "Dark matter in galaxies," Pub. Astron. Soc. Pac., 104, pp. 1109-138, 1992.

2. Alcock, C. et al., "The MACHO project large-magellanic-cloud microlensing results from the first 2 years and the nature of the galactic dark halo," Astrop. J., 486, pp. 697-726, 1997.

3. Burrows, A. and Liebert, J., “Astronomy - Probing Dark Matter,” Nature 373, pp. 191-192, 1995.

4. Matthews, K., Nakajima, T., Kulkarni, S.R., and Oppenheimer, B.R., "Spectral energy-distribution and bolometric luminosity of the cool brown dwarf gliese 229B," Astron. J., 112, pp. 1678-1682, 1996.

5. Saumon, D., Bergeron, P., Lunine, J.I., Hubbard, W.B., and Burrows, A., "Cool Zero-Metallicity Stellar Atmospheres," Astrop. J., 424, pp. 333-344, 1994.

6. Adam, F.C. and Laughlin, G., "Implications of white dwarf galactic halos," Astrop. J., 468, pp. 586-597, 1996.

7. Boughn, S.P., Saulson, P.R., and Seldner, M., "A new limit on the mass-to-light ratio of the halo of NGC 4565," Astrop. J., 250, L15-L19, 1981.

8. Skrutskie, M. F., Shure, M. A. \& Beckwith, S. 1985, "Faint photometry of edge-on spiral galaxies - a search for massive halos," Astrop. J., 299, pp. 303-311, 1985.

9. Sackett, P.D., Morrison, H.L., Harding, P., and Boroson, T.A., "A faint luminous halo that may trace the dark matter around spiral galaxy NGC 5907," Nature, 370, pp. 441-443, 1994.

10. Lequeux, J., Fort, B., Dantel-Fort, M., Cuillandre, J.-C., and Mellier, Y., "V-band and I-band observations of the halo of NGC 5907," Astron. \& Astrop., 312, pp. L1-L4, 1996.

11. Cowie, L.L., Gardner, J.P., Lilly, S.J., and McLean, I., “A K-band deep galaxy survey,” Astrop. J., 360, pp. L1-L5, 1990.

12. Hauser, M. G. 1996, in IAU Symp. 168, eds. M. Kafatos and Y. Kondo, Dordrecht: Kluwer, 99.

13. Franceschini, A., Toffolatti, L., Mazzei, P., Danese, L., Dezotti, G., "Galaxy counts and contributions to the background radiation from $1 \mu \mathrm{m}$ to $1000 \mu \mathrm{m}, " A \& A S, 89$, pp. 285-310, 1991.

14. Pipher, J.L., Forrest, W.J., and Wu, J., "InSb Arrays for SIRTF," SPIE Proc., 2475, pp. 428-434, 1995.

15. Wildeman, K.J., Ploeger, G.R., Beintema, D.A., "Shutter for the short wavelength spectrometer in ISO," Cryogenics, 29, pp. 546-549, 1989.

16. Uemizu, K. et al, in preparation. 